\title{
Comparison and Analysis of Self-Reference Image with Meaningful Image for Robust Watermarking Algorithm based on Visual Quality and Fidelity
}

\author{
S.M.Ramesh \\ Sr.Asst.Professor \\ Bannari Amman Institute of \\ Technology, Sathy-638401 \\ INDIA.
}

\author{
Dr.A.Shanmugam \\ Professor \\ Bannari Amman Institute of \\ Technology, Sathy-638401 \\ INDIA.
}

\author{
B.Gomathy \\ Asst.Professor \\ Bannari Amman Institute of \\ Technology, Sathy-638401 \\ INDIA.
}

\begin{abstract}
This paper presents a comparison and analysis of recently developed watermarking algorithms. Digital watermarking has been regarded as a vital technique for safeguarding the copyright of digital contents. A digital watermarking method is said to be effective if the watermark embedded in an image by it could survive against diverse attacks ranging from compression, filtering to cropping. The performance of the watermarking algorithms is analyzed and to estimate and compare robustness of watermarking algorithms by considering the visual quality of the original and watermarked images in terms of Peak Signal to Noise Ratio. Furthermore, the extracting fidelity of the watermarking algorithms is compared by taking the Normalized Correlation value between the original watermark and the extracted watermark. The experimental results showed the accuracy of different watermarking algorithms in terms of visual quality and fidelity.
\end{abstract}

\section{Keywords}

Robust Watermarking, Image Compression, Wavelet Transform, PSNR (Peak Signal to Noise Ratio), NC (Normalized Correlation).

\section{INTRODUCTION}

Digital watermarking is a technique used to protect digital images from illegal copying and exploitation. Data is embedded into a multimedia element like image, audio or video by the process of watermarking. Diverse applications such as copyright protection, access control, and broadcast monitoring use this embedded data extracted or detected from the multimedia element [4]. Digital watermarking has attracted the attention of a considerable number of researchers in the period between early to mid-1990s. From that time onwards the number of publications per year has increased rapidly to several hundreds [1]. It began from simple approaches offering the basic principles to highly-developed communication theory results oriented algorithms and applying them for the watermarking problem [7],[8],[9],[12] and [13]. Cover image, watermark structure, embedding algorithm, and extraction or detection algorithm are the basic components of an image watermarking algorithm [15].

The following requirements must be fulfilled by a good watermarking system [3]: Transparency: The degree of degradation of the quality of the quality due to the embedded watermark must be minimal. Robustness: Common image processing operations such as cropping, rotation, filtering and compression should not affect the embedded watermark [6], [2] and [10]. Security: Even if the embedding algorithm is made public, the watermarking scheme must be secure. Cryptographic techniques are commonly employed to accomplish security. Appropriate complexity: The compression/decompression processes should require small amount of computation and memory, particularly for real time application. Careful design is required for tradeoff because it is evident that these requirements may conflict with each other. In general, Watermarks and watermarking methods can be classified into different categories in several ways. Digital watermarking can be classified into image watermarking, video watermarking and audio watermarking [5] on the basis of the range of application. Watermarking techniques are broadly classified into spatialdomain and frequency-domain methods [14] depending on the domain in which the watermark is inserted.

\section{PREVIOUS WORK}

A handful of watermarking schemes have been presented in the literature for protecting the copyrights of digital images. Recently, robust watermarking schemes have received a great deal of attention among watermarking researchers because of its performance and effectiveness. A brief review of some recent researches is presented here.

Yu-Ting Pai et al. [16] have combined the benefits of blockbased permutation with that of neighboring coefficient embedding and proposed a high quality and robust watermarking algorithm. In order to hide more information into high frequency blocks without causing severe distortion to the watermarked image, the proposed approach has used the relationship between the coefficients of neighboring blocks. Also, robustness to the mid-frequency filter attacks has been improved by a proposed extraction method. The experimental outcomes have shown that, the peak signal to noise ratio (PSNR) has been increased by the proposed approach to achieve highly effective perceptual visibility. Also, the proposed approach has been robust to several signal processing operations, like compression (JPEG), image cropping, sharpening, blurring, and brightness adjustments. The robustness of their proposed technique particularly for blurring attack has been evident from their experimentations.

Amit Bohra et al. [17] have proposed a technique based on second-generation wavelets (lifting-based integer wavelets transform) for robust watermarking of images. The capability of blind self-authentication of the watermarked image has been 
present in the proposed approach along with robustness. Due to the employment of integer-to-integer transform, the watermarked images have not shown any perpetual degradation and a peak signal to noise ratio (PSNR) in excess of $40 \mathrm{~dB}$ has been obtained. For diverse attacks for example filtering, compression and rotation, the proposed technique has shown superior performance over other similar existing schemes, in the simulation results. Also, alterations in the image generates dissimilar sequences because of the two independent processes that has been employed in the proposed watermark sequence extraction, thereby alterations in the image have been detected.

Wei $\mathrm{Lu}$ et al. [19] have presented a robust, feature point detection and image normalization based watermarking scheme. First, using the proposed multi-resolution feature point detection filter, they have identified some stable feature points from the original image. Then, image normalization has been performed on the disks centered at these feature points. For each disk the watermark has been embedded separately in the sub band coefficients of DFT domain. The original image has not been required and the correlation between the watermark embedding coefficients and the original watermark has been used by the watermark detection process. The proposed scheme which achieved strong robustness to signal processing and geometrical distortions has combined the advantages of feature point detection and image normalization. The superior performance of the proposed scheme has been demonstrated by experimental results.

\section{WATERMARKING ALGORITHMS}

The main objective of the proposed research is to compare the watermarking algorithms described in the literature [6], [11], [18] and [20]. Algorithms proposed to perform robust watermarking in the frequency domain are selected for comparison. Their robustness is obtained for several compression standards and the results are extensively analyzed for diverse compression ratios. The following subsections provide a detailed description of the embedding and extracting process of the watermarking algorithms.

\subsection{Liu J.L et al. Algorithm [6]}

Liu J.L et al. [6] have proposed a self-reference image based robust watermarking scheme. In order to overcome the weak robustness problem in spatial domain, their robust watermarking scheme has modified the original image in transform domain and has embedded the watermark in the difference values between the original image and its reference image. Furthermore, the application has been more practical in real life application for ownership verification because original images have not been required for watermark extraction.

\subsubsection{Watermark embedding steps}

Step 1: Using one-level wavelet transform, transform the original image into wavelet coefficients.

Step 2: Set the three high-frequency sub-bands $\left(\mathrm{LH}_{1}, \mathrm{HL}_{1}\right.$ and $\left.\mathrm{HH}_{1}\right)$ to zero.

Step 3: Determine its reference image by applying inverse wavelet transform.

Step 4: Calculate the differences between the original image $\mathrm{W}$ and its reference image $\mathrm{W}^{1}$, and determine the location $i d w(i, j)$ such that

$$
s<\mid w(i, j)-w^{\prime}(i, j)<t
$$

where $s$ and $t \in Z^{+}$.

Step 5: Select some locations at random for embedding on the basis of the result of Step 4. Embed the watermark is the visual weight to balance the tradeoff between the robustness and imperceptibility.

The block diagram of the watermark embedding process is following fig. 1 .

\subsubsection{Watermark extraction steps}

Step 1: Using one-level wavelet transform, transform the watermarked image into wavelet coefficients.

Step 2: Set the three high-frequency sub-bands $\left(\mathrm{LH}_{1}, \mathrm{HL}_{1}\right.$ and $\mathrm{HH}_{1}$ ) to zero.

Step 3: Determine its reference image $W^{\prime}{ }_{x}$ by performing inverse wavelet transform.

Step 4: Determine the embedded watermark bits according to the sequence of embedding location, as follows,

$$
x^{\prime}(k)=\left\{\begin{array}{l}
1 \text { if } \mathrm{w}_{\mathrm{x}}(i d w(i, j)) \geq w_{x}^{\prime}(i d w(i, j)) \\
-1 \text { if } \mathrm{w}_{\mathrm{x}}(i d w(i, j))<w_{x}^{\prime}(i d w(i, j))
\end{array}\right. \text {-- (2) }
$$

The block diagram of the watermark extraction process is following fig. 2 .

\subsection{Ye J.X and Tan. G Algorithm [11]}

Ye.J.X and Tan.G [11] have proposed an improved digital watermarking algorithm for a meaningful image. They have processed the image using Arnold transformation and correlation detection and multiplied the spectrum of the watermarking with the pseudo-random series to obtain the private key.

\subsubsection{Watermark embedding steps}

Step 1: Divide the original image into $8 \times 8$ image blocks.

Step 2: For each sub-block image, execute FFT translation after performing DFT transformation. Make DC element in the middle by exchanging the first quadrant and the third quadrant of the two-dimensional matrix with the second quadrant and the fourth quadrant respectively.

Step 3: Using the Arnold transformation, the binary-value image watermark is scrambled.

Step 4: Generate two uncorrelated pseudo-random sequences A and $\mathrm{B}$, In order to generate identical pseudo-random sequence, use the same key for embedding the watermark and extracting the watermark.

Step 5: Multiply the pseudo-random sequence A by the corresponding elements of spectrum if the value of the watermark matrix is 0 , otherwise multiply the other pseudo-random sequence B by the corresponding elements of spectrum.

Step 6: Obtain the image with watermark, by executing $I D F T$ transformation for each sub-block image.

The block diagram of the watermark embedding process is following fig. 3 .

\subsubsection{Watermark extraction steps}

Step 1: Generate $8 \times 8$ sub-block images by partitioning the embedded watermark image. 
Step 2: Execute the DFT transformation for each sub-block image.

Step3: Using the same key as the embedding operation generate two uncorrelated pseudo-random sequences A and B same as the embedding operation

Step 4: If the spectrum of the embedded watermark is more relative with $\mathrm{A}$ than $\mathrm{B}$, the point of image is embedded with 0 of the watermark matrix. Otherwise, the point of image is embedded with 1 of the watermark matrix.

Step 5: Obtain the watermark image after scrambling the

watermark matrix by the Arnold transformation.

The block diagram of the watermark extraction process is following fig. 4 .

\section{EXPERIMENTAL RESULTS COMPARATIVE ANALYSIS}

This section describes the experimental results and comparative analysis of four considered algorithms. These algorithms are implemented using MATLAB. Here, for comparison, we have taken the watermark image shown in following figures and the host images such as, lena, baboon and cameraman. For each input host images, the watermark image is embedded based on embedding algorithms and the watermark image is extracted from the watermarked image using extraction algorithms. The following diagram shows the watermarked image and extracted watermark of the considered algorithms for the taken input images. Then, the outputs obtained from the different watermarking algorithms are compared based on the metrics, for example, PSNR and NC. The visual quality is evaluated for the algorithms by the peak signal-to-noise ratio (PSNR) criterion in between the host images with the watermarked images and the extracting fidelity is compared by the Normalized Correlation (NC) value between the original watermark and the extracted watermark. The definition of PSNR and NC is given in the following equations.

$$
P S N R=10 \log _{10} \frac{E_{\max }^{2} \times I_{w} \times I_{h}}{\sum\left(I_{x y}-I_{x y}^{*}\right)}
$$

Where, $I_{w}$ and $I_{h} \rightarrow$ Width and height of the watermarked

image. $I_{x y} \rightarrow$ Original image pixel value at coordinate $(x, y)$.

$I_{x y}^{*} \rightarrow$ Watermarked image pixel value at coordinate $(x, y)$.

$E_{\max }^{2} \rightarrow$ Largest energy of the image pixels (i.e., $E_{\max }=255$

for 256 gray-level images).

$$
N C=\frac{\sum_{i=0}^{N} \sum_{j=0}^{M} W(i, j) \times W^{*}(i, j)}{\sum_{i=0}^{N} \sum_{j=0}^{M}[W(i, j)]^{2}}
$$

Where, $W(i, j) \rightarrow$ Original watermark image

$W *(i, j) \rightarrow$ Extracted watermark image

$N$ and $M \rightarrow$ Width and height of the watermark image

The figures shown in Table 1 and 2 are intermediate images obtained by applying the watermarking algorithms employed in the proposed work. For each host images, the results are taken and their corresponding PSNR and NC are computed.

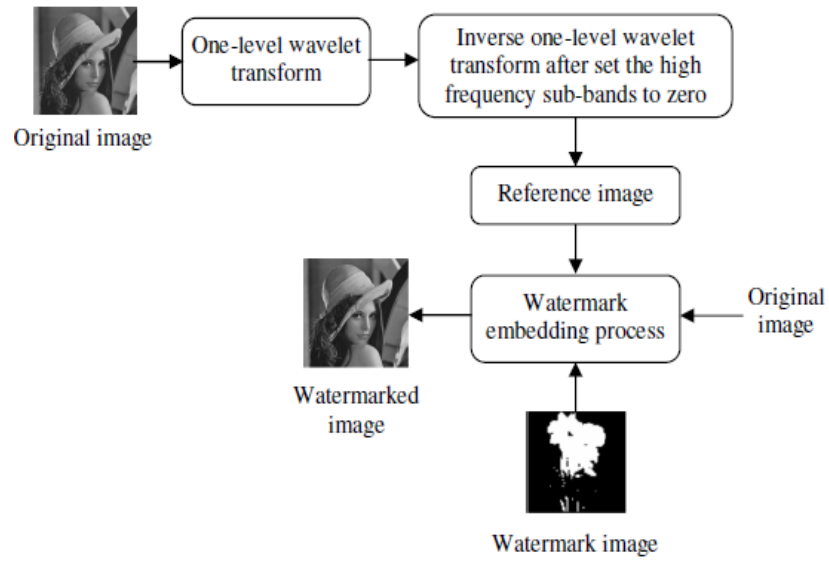

Fig 1: Watermark embedding process

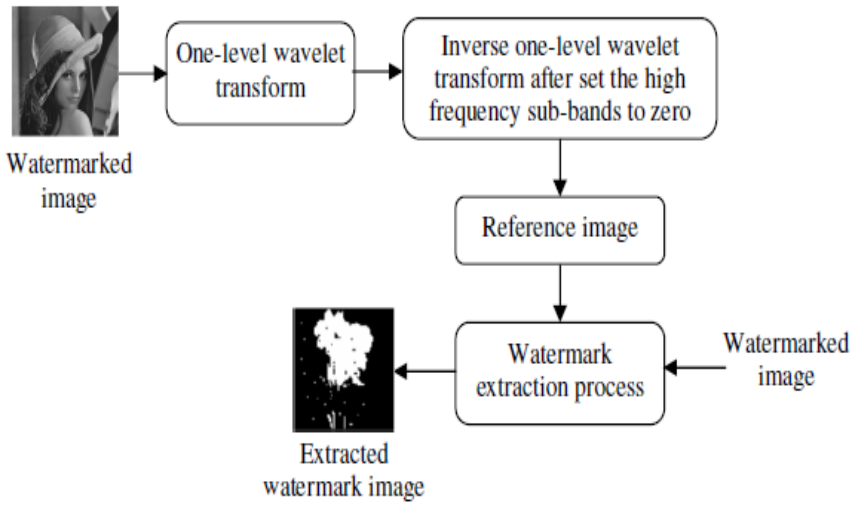

Fig 2: Watermark extraction process. 
Table 1. Liu J.L et al. algorithm

\begin{tabular}{|c|c|c|c|c|c|c|}
\hline & Input Image & $\begin{array}{c}\text { Watermark } \\
\text { Image }\end{array}$ & $\begin{array}{c}\text { Watermarked } \\
\text { Image }\end{array}$ & $\begin{array}{c}\text { Extracted } \\
\text { Watermark } \\
\text { Image }\end{array}$ & PSNR & NC \\
\hline Lena & & & & & 51.8779 & 0.9927 \\
\hline Baboon & & & & & 48.8827 & 0.9893 \\
\hline Cameraman & & & & & 52.5641 & 0.9927 \\
\hline
\end{tabular}

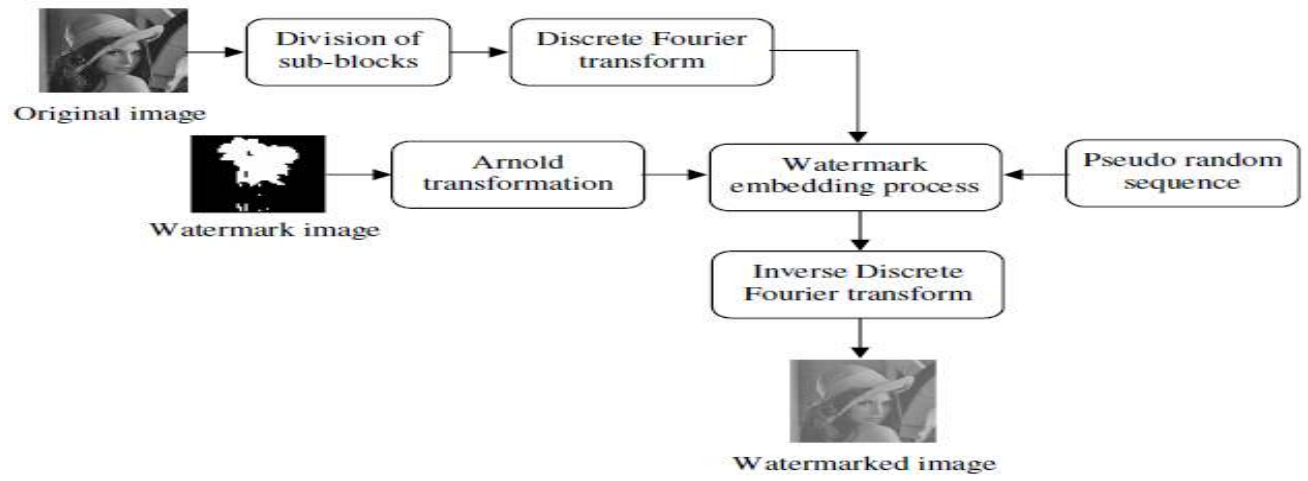

Fig 3: Watermark embedding process.

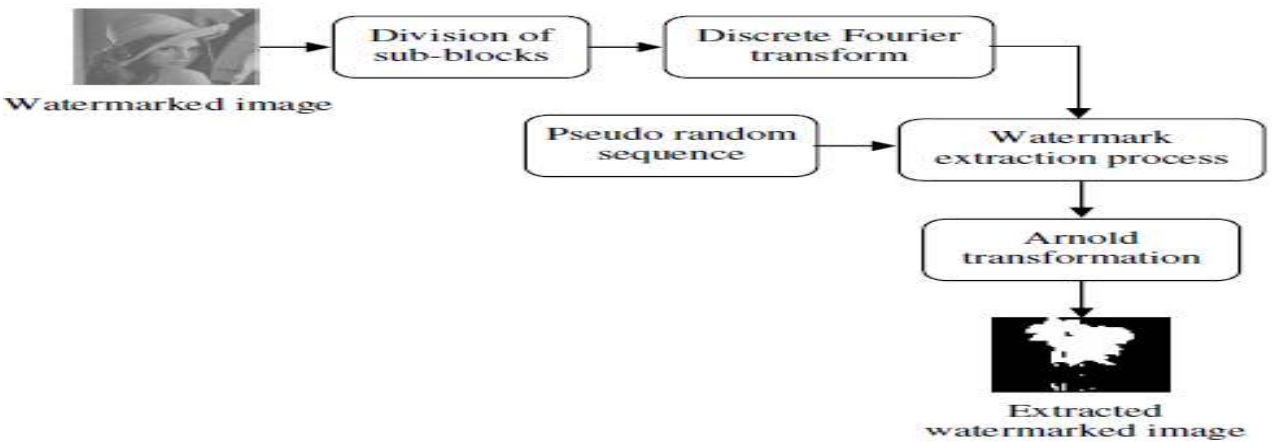

Fig 4: Watermark extraction process. 
Table 2. Ye J.X and Tan. G Algorithm

\begin{tabular}{|c|c|c|c|c|c|c|}
\hline & Input Image & $\begin{array}{c}\text { Watermark } \\
\text { Image }\end{array}$ & $\begin{array}{c}\text { Watermarked } \\
\text { Image }\end{array}$ & $\begin{array}{c}\text { Extracted } \\
\text { Watermark } \\
\text { Image }\end{array}$ & PSNR & NC \\
\hline Lena & & & & & 51.6707 & 1 \\
\hline Baboon & & & & & 38.0392 & 1 \\
\hline Cameraman & & & & & 48.0703 & 1 \\
\hline
\end{tabular}

\section{CONCLUSION}

In this paper, an extensive analysis of two robust watermarking algorithms done in the frequency domain. The performance study was carried out on two watermarking algorithms in terms of visual quality and the fidelity. The comparative study has revealed that Liu J.L et al's algorithm achieved better visual quality when compared with other algorithms. Moreover, the experimental results have shown that Liu J.L et al's algorithm was better (in terms of fidelity) of the other algorithms. In our future work, we will investigate in embedding multiple watermarks in $\mathrm{D}$ and $\mathrm{U}$ matrices so that the watermark image can survive to more number of image attacks.

\section{REFERENCES}

[1] F.A.P. Petitcolas, R.J. Anderson, M.G. Kuhn, "Information hiding-a survey," Proc. IEEE Vol: 87, No: 7, pp: 10621078, 1999.

[2] Shelby Pereia and Thierry Pun, "Fast Robust Template Matching for Affine Resistant Image Watermarks," Proceedings of the Third International Workshop on Information Hiding, Lecture Notes in Computer Science (LNCS), Vol. 1768, pp. 199 - 210, 1999.

[3] G. C. Langelaar, I. Setywan, and R. L. Lagendijk, "Watermarking digital image and video data," IEEE Signal Process. Mag., Vol. 17, No. 5, pp. 20-46, Sep. 2000.
[4] Cox I. J., Miller, M. L. and Bloom J. A., "Digital Watermarking", Morgan Kaufmann Publishers, USA, 2002.

[5] Xiang-Yang Wang and Hong Zhao, "A Novel Synchronization Invariant Audio Watermarking Scheme Based on DWT and DCT", IEEE Transactions On Signal Processing, Vol. 54, No. 12, December 2006.

[6] Jiang-Lung Liu, Der-Chyuan Lou, Ming-Chang Chang and Hao-Kuan Tso, "A robust watermarking scheme using selfreference image", Computer Standards \& Interfaces, Vol. 28, no. 3, pp.356-367, January 2006.

[7] Lino Coria, Panos Nasiopoulos, Rabab Ward and Mark Pickering. "An Access Control Video Watermarking Method that is Robust to Geometric Distortions", Journal of Information Assurance and Security, Vol: 2, pp: 266274, 2007.

[8] Krzysztof Szczypiorski, Igor Margasinski, Wojciech Mazurczyk. "Steganographic Routing in Multi Agent System Environment", Journal of Information Assurance and Security, Vol: 2, pp: 235-243, 2007.

[9] Zbigniew Piotrowski, Piotr Gajewski. "Voice spoofing as an impersonation attack and the way of protection", Journal of Information Assurance and Security, Vol: 2, pp: 223-225, 2007.

[10] Grace C.-W. Ting, Bok-Min Goi and Swee-Huay Heng, "Attacks on a robust watermarking scheme based on self- 
reference image", Computer Standards \& Interfaces, Vol. 30, No. 1-2, pp. 32-35, January 2008.

[11] Ji Xiang Ye, Guan Zheng Tan, "An Improved Digital Watermarking Algorithm for Meaningful Image", in proceedings of International Conference on Computer Science and Software Engineering, pp. 822 - 825, Wuhan, Hubei, 2008.

[12] B Surekha, Dr GN Swamy, Dr K Srinivasa Rao, A Ravi Kumar. "A Watermarking Technique based on Visual Cryptography", Journal of Information Assurance and Security, Vol: 4, pp: 470-473, 2009.

[13] Zorana Bankovic, José M. Moya, Álvaro Araujo, Slobodan Bojanic and Octavio Nieto-Taladriz. "A Genetic Algorithm-based Solution for Intrusion Detection”,, Journal of Information Assurance and Security, Vol: 4, pp: 192199, 2009.

[14] Sanjeev kumar, Balasubramanian Raman, Manoj Thakur, "Real coded genetic algorithm based stereo image watermarking", IJSDIA Intl. Journal of secure digital information Age, Vol:1, No:1, 2009.

[15] Chih-Chin Lai, Hsiang-Cheh Huang and Cheng-Chih Tsai, "A Digital Watermarking Scheme Based On Singular Value Decomposition And Micro-Genetic Algorithm", International Journal of Innovative Computing, Information and Control, Vol. 5, No. 7, July 2009.

[16] Yu-Ting Pai, Shanq-Jang Ruan and Jurgen Gotze, "A High Quality Robust Watermarking Scheme", Lecture Notes in Computer Science, Vol. 4261, pp. 650-657, 2006.

[17] Amit Bohra, Omar Farooq and Izharuddin, "Blind selfauthentication of images for robust watermarking using integer wavelet transform", AEU - International Journal of Electronics and Communications, Vol. 63, No. 8, pp. 703707, August 2009.

[18] Chih-Ming Kung, Shu-Tsung Chao, Yen-Chen Tu, Yu-Hua Yan, Chih-Hsien Kung, "A Robust Watermarking and Image Authentication Scheme used for Digital Contant Application", Journal of Multimedia, Vol 4, No 3, pp. 112119, June 2009.

[19] Wei Lu, Hongtao Lu and Fu-Lai Chung, "Feature based robust watermarking using image normalization", Computers \& Electrical Engineering, Vol. 36, No. 1, pp. 218, January 2010.

[20] Shinfeng D. Lin, Shih-Chieh Shie and J.Y. Guo, "Improving the robustness of DCT-based image watermarking against JPEG compression", Computer Standards \& Interfaces, Vol. 32, no. 1-2, pp. 54-60, January 2010 .

\section{AUTHORS PROFILE}

S.M.Ramesh received the B.E degree in Electronics and Communication Engineering from National Institute of Technology (Formerly Regional Engineering College), Trichy, Bharathidhasan University, India in the year 2001 and the M.E, degree in Applied Electronics from R.V.S. College of Engineering and Technology, Dindugal, Anna University, India in the year 2004. From 2004 to 2005, he served as a Lecturer in the Department of Electronics and Communication Engineering, Maharaja Engineering College, Coimbatore, India. From 2005 to 2006, he served as a Lecturer in the Department of Electronics and Communication Engineering, Nandha Engineering College, Erode, India. Since June 2006, he served as Senior Lecturer, in the Department of Electronics and Communication Engineering Bannari Amman Institute of Technology, Sathyamangalam, Erode, India. He is currently Pursuing the Ph.D. research work in Anna University-Chennai. His current research focuses on Image Processing for Compression, Segmentation-VLSI Design for low voltage and Soft Computing in Fuzzy. He is working very closely with Prof. Dr.A.Shanmugam , Prof Dr.R.Harikumar and Prof Dr.S.Valarmathy.

Dr.A.Shanmugam received the BE Degree in PSG College of Technology in 1972, Coimbatore and ME Degree from College of Engineering, Guindy, Chennai in 1978 and Doctor of Philosophy in Electrical Engineering from Bharathiar University, Coimbatore in 1994.From 1972-76, he worked as Testing Engineer in Testing and Development Centre, Chennai. He was working as a Lecturer Annamalai University in 1978. $\mathrm{He}$ was the Professor and Head of Electronics and Communication Engineering Department at PSG College of Technology, Coimbatore during 1999 to 2004. Authored a book titled "Computer Communication Networks" which is published by ISTE, New Delhi, 2000.He is currently the Principal, Bannari Amman Institute of Technology, Sathyamangalam..He is on the editorial board of International Journal Artificial Intelligence in Engineering \& Technology (ICAIET), University of Malaysia, International Journal on "Systemics, Cybernetics and Informatics (IJSCI)" Pentagram Research Centre, Hyderabad, India. He is member of the IEEE, the IEEE computer society. 